\title{
Linearization and input-output decoupling for general nonlinear systems
}

\section{A.J. van der SCHAFT \\ Department of Applied Mathematics, Twente University of Tech- nology, P.O. Box 217, 7500 AE Enschede, the Netherlands}

Received 16 March 1984

Revised 31 May 1984

Necessary and sufficient conditions are obtained for the linearization and input decoupling (by state feedback) of general nonlinear systems. It is shown how these conditions can be derived from the already known conditions for affine nonlinear systems, thereby also elucidating the existing theory for affine systems.

AMS(MOS) Subject Classification: 93C10, 93B10, 53B15.

Keywords: Linearization, Input-output decoupling, Extended system, Fibre bundles, Horizontal distributions.

\section{Introduction}

This paper is concerned with the generalization of some recently obtained results for nonlinear systems of the form

$\dot{x}=A(x)+\sum_{i=1}^{m} u_{i} B_{i}(x)$

(such systems are called affine, or input-linear) to general nonlinear systems

$\dot{x}=f(x, u)$.

As such the paper is a continuation of [7], where the necessary and sufficient conditions for (local) controlled invariance for affine systems were generalized to general nonlinear systems, thereby solving for instance the disturbance decoupling problem for these systems (see [8]). First we recall the framework used in these references.

A coordinate free description of a smooth nonlinear system (1.2) consists of the following ingredients (see [7,12] for references). The state space $M$ is a smooth manifold of dimension $n$ (smooth will always mean $C^{\infty}$ or $C^{k}$ with $k$ sufficiently large). There is a smooth $(n+m)$-dimensional fibre bundle $B$ over $M$, with projection $\pi: B \rightarrow M$. Finally a smooth map $f: B \rightarrow T M$ is given such that the diagram

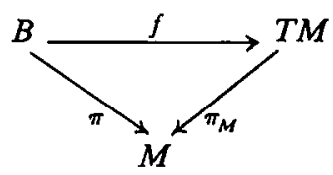

commutes $\left(\pi_{M}\right.$ is the usual projection of $T M$ on $M)$. We denote the system (1.3) by $\Sigma(M, B, f)$. Take local coordinates

$(x, u)=\left(x_{1}, \ldots, x_{n}, u_{1}, \ldots, u_{m}\right)$

for $B$ such that $\left(x_{1}, \ldots, x_{n}\right)$ are coordinates for $M$ $((x, y)$ are called fibre respecting coordinates). In such coordinates $(x, u)$ for $B$ and $(x, \dot{x})$ for $T M$, we can write by the commutativity of (1.3) and with a slight abuse of notation

$f(x, u)=(x, f(x, u))$,

and one immediately recovers the local coordinate expression (1.2). In the case of an affine system (1.1), $B$ is a vector bundle and $f: B \rightarrow T M$ is an affine map. Hence the image of $f$ is an affine distribution $\Delta$ on $M$, in local coordinates given by

$\Delta(x)=A(x)+\Delta_{0}(x)$

with $\Delta_{0}$ the distribution

$\Delta_{0}(x)=\operatorname{span}\left\{B_{1}(x), \ldots, B_{m}(x)\right\}$,

for certain (locally) defined vector fields $A$, $B_{1}, \ldots, B_{m}$ on $M$. We denote the affine system also by $\left(\Delta, \Delta_{0}\right)$.

Of fundamental importance to us is the fact that we can associate with every nonlinear system $\Sigma(M, B, f)$ an affine system $\left(\Delta^{\mathrm{e}}, \Delta_{0}^{\mathrm{e}}\right)$, the extended system, introduced in [12]. The affine distribution $\Delta^{e}$ on $B$ is defined as

$\Delta^{\mathrm{e}}(x, u)=\left\{X \in T_{(x, u)} B \mid \pi_{*} X=f(x, u)\right\}$ 
and the distribution $\Delta_{0}^{\mathrm{e}}$ as

$\Delta_{0}^{\mathrm{e}}(x, u)=\left\{X \in T_{(x, u)} B \mid \pi_{*} X=0\right\}$.

If $\Sigma(M, B, f)$ is in local fibre respecting coordinates given by $\dot{x}=f(x, u)$, then $\left(\Delta^{\mathrm{e}}, \Delta_{0}^{\mathrm{e}}\right)$ is given by

$\dot{x}=f(x, u), \quad \dot{u}=v$,

with $v \in \mathbb{R}^{m}$ the new input. Therefore, taking the extended system amounts to integrating the inputs one time'.

\section{Controlled invariance}

First we quickly review the results on (local) controlled invariance for general nonlinear systems $\Sigma(M, B, f)$ obtained in [7], see also [13]. The concept of controlled invariance for nonlinear systems was firstly introduced in $[1,3]$. We call a distribution regular if it is involutive and has constant dimension. Then a regular distribution $D$ on $M$ is called locally controlled invariant if for every $x \in M$ there exists an open neighborhood $V$ of $x$ and a trivialization $\pi^{-1}(V) \simeq V \times U$ (U) is the standard fibre of $B$ ) such that

$[f(\cdot, u), D] \subset D, \quad \forall u \in U$,

with

$f: V \times U=\pi^{-1}(V) \rightarrow T V$.

In other words there exist local fibre respecting coordinates $(x, u)$ such that in these coordinates $[f(\cdot, u), D] \subset D$ for every $u$. Given a set of fibre respecting coordinates $(x, u)$ for $B$ and a corresponding representation $\dot{x}=f(x, u)$ this is equivalent to the local existence of a nonlinear feedback

$u=\alpha(x, \tilde{u})$

with $(\partial \alpha / \partial \tilde{u})$ everywhere nonsingular, such that $[\tilde{f}(\cdot, \tilde{u}), D] \subset D$ for every $\tilde{u}$, where $\dot{x}=\tilde{f}(x, \tilde{u})$ is the feedback transformed system, i.e.

$\tilde{f}(x, \tilde{u})=f(x, \alpha(x, \tilde{u}))$.

The basic idea is that local feedback corresponds to another choice of fibre respecting coordinates $(x, \tilde{u})$.

Necessary and sufficient conditions for local controlled invariance of $D$ are now given as follows. Since $D$ is regular, there exist local coordi- nates $x=\left(x_{1}, \ldots, x_{n}\right)$ such that

$D=\operatorname{span}\left\{\frac{\partial}{\partial x_{1}}, \ldots, \frac{\partial}{\partial x_{k}}\right\} \quad(k \leqslant n)$.

Then we define the regular distribution $\dot{D}$ on $T M$ by setting $[7,13]$

$\dot{D}=\operatorname{span}\left\{\frac{\partial}{\partial x_{1}}, \ldots, \frac{\partial}{\partial x_{k}}, \frac{\partial}{\partial \dot{x}_{1}}, \ldots, \frac{\partial}{\partial \dot{x}_{k}}\right\}$

with the coordinate functions $\dot{x}_{i}$ on $T M$ defined by $\dot{x}_{i}(v)=\mathrm{d} x_{i}(v), \quad v \in T M$.

Theorem 2.1 [7]. Let $\Sigma(M, B, f)$ be a nonlinear system, let $D$ be a regular distribution on $M$ and assume that the distribution $\Delta_{0}^{\mathrm{e}} \cap f^{-1}(\dot{D})$ has constant dimension (with $\Delta_{0}^{\mathrm{e}}$ as in (1.5)). Then $D$ is locally controlled invariant if and only if

$f_{*}\left(\pi_{*}^{-1}(D)\right) \subset \dot{D}+f_{*}\left(\Delta_{0}^{\mathrm{e}}\right)$.

Let now $D$ be regular, locally controlled invariant and such that $\Delta_{0}^{e} \cap f_{*}^{1}(\dot{D})$ has constant dimension. Then it is quite easily seen that $E=$ $f_{*}^{1}(D)$ is a regular distribution on $B$ such that $\pi_{*} E=D$ and $f_{*} E \subset \dot{D}$ (see [13]). This is also equivalent to

$\left[\Delta^{\mathrm{e}}, E\right] \subset E+\Delta_{0}^{\mathrm{e}}, \quad \pi_{*} E=D$.

On the other hand, a regular distribution $E$ on $B$ such that $E \cap \Delta_{0}^{\mathrm{e}}$ has constant dimension is locally controlled invariant if and only if

$\left[\Delta^{\mathrm{e}}, E\right] \subset E+\Delta_{0}^{\mathrm{e}}$

(see $[3,7])$. Therefore $E=f_{*}^{-1}(\dot{D})$ is locally controlled invariant w.r.t. $\left(\Delta^{\mathrm{e}}, \Delta_{0}^{\mathrm{e}}\right)$. Conversely if $E$ is a regular distribution on $B$ (with $E \cap \Delta_{0}^{e}$ of constant dimension), which is locally controlled invariant w.r.t. $\left(\Delta^{\mathrm{e}}, \Delta_{0}^{\mathrm{e}}\right)$, then $\pi_{*} E$ is well defined [12] and is a locally controlled invariant distribution w.r.t. $\Sigma(M, B, f)$. This shows the close relationship between local controlled invariance w.r.t. $\Sigma(M, B, f)$ and w.r.t. the extended system $\left(\Delta^{\mathrm{e}}, \Delta_{0}^{\mathrm{e}}\right)$. In terms of the required feedbacks we obtain the following. Let $D$ be locally controlled invariant w.r.t. $\Sigma(M, B, f)$ and let $E$, with $\pi_{*} E=$ $D$, be locally controlled invariant w.r.t. $\left(\Delta^{\mathrm{e}}, \Delta_{0}^{\mathrm{e}}\right)$. Suppose that the feedback $u=\alpha(x, \tilde{u})$ makes $D$ invariant, i.e. $[\vec{f}(\cdot, u), D] \subset D$. Let us formally 
differentiate $\alpha(x, \tilde{u})$ along $\dot{x}=f(x, u)$ :

$$
\frac{\mathrm{d}}{\mathrm{d} t} \alpha(x, \tilde{u})=\frac{\partial \alpha(x, \tilde{u})}{\partial x} f(x, u)+\frac{\partial \alpha(x, \tilde{u})}{\partial \tilde{u}} \dot{\dot{u}} .
$$

Then it is easily seen that the affine feedback

$v=\frac{\partial \alpha(x, \tilde{u})}{\partial x} f(x, u)+\frac{\partial \alpha(x, \tilde{u})}{\partial \tilde{u}} \tilde{v}$

with $\tilde{v} \in \mathbb{R}^{m}$ the new input, and where we substitute (after performing the differentiations) $\tilde{u}=$ $\beta(x, u)$ with $\beta$ satisfying $\alpha(x, \beta(x, u))=u$, is the required feedback which makes $E$ invariant. This was first noted, in the case of the extended system of an affine system, in [1]. Consequently the required feedback for the extended system can always be chosen in the special form (2.8).

\section{Linearization}

First we will review some results about the linearization by feedback of an affine system $\left(\Delta, \Delta_{0}\right)$ with state space $M$. Let us define the following sequence of distributions $\Delta_{i}$ on $M$ :

$\Delta_{1}=\left[\Delta, \Delta_{0}\right]$,

$\Delta_{i+1}=\left[\Delta, \Delta_{i}\right], \quad i \geqslant 1$.

It is clear that $\Delta_{0} \subset \Delta_{1} \subset \Delta_{2} \subset \cdots$. The main theorem is now as follows $[2,4,14]$.

Theorem 3.1. Let $x_{0} \in M$ be such that $\Delta\left(x_{0}\right)=$ $\Delta_{0}\left(x_{0}\right)$. Then there exist coordinates $x$ around $x_{0}$ such that $\left(\Delta, \Delta_{0}\right)$ has the controllable linear representation

$\dot{x}=A x+B u$

(i.e. $\Delta(x)=A x+\operatorname{Im} B$ with $A$ and $B$ matrices) if and only if the distributions $\Delta_{i}$ are regular in $a$ neighborhood of $x_{0}$ and $\operatorname{dim} \Delta_{n-1}\left(x_{0}\right)=n \quad(=$ $\operatorname{dim} M$ ).

Remark. If the affine system $\left(\Delta, \Delta_{0}\right)$ has a representation

$\dot{\tilde{x}}=A(\tilde{x})+\sum_{i=1}^{m} \tilde{u}_{i} B_{i}(\tilde{x}), \quad A(0)=0$,

then this is equivalent to the existence of a feed- back

$\tilde{u}=\alpha(\tilde{x})+\beta(\tilde{x}) u$,

with $\beta(\tilde{x})$ an $m \times m$-matrix, and a coordinate transformation

$\tilde{x}=\phi(x), \quad$ with $\phi(0)=0$,

such that (3.3) is transformed into (3.2).

Now we want to derive the conditions in order to transform (locally) a general nonlinear system $\dot{x}=f(x, u)$ by a coordinate transformation and a general nonlinear feedback $u=\alpha(x, \tilde{u})$ into a linear system. Let therefore $\Sigma(M, B, f)$ be a nonlinear system with extended system $\left(\Delta^{\mathrm{e}}, \Delta_{0}^{\mathrm{e}}\right)$. Define the distribution $\Delta_{i}^{\mathrm{e}}$ on $B$ by

$\Delta_{i+1}^{\mathrm{e}}=\left[\Delta^{\mathrm{e}}, \Delta_{i}^{\mathrm{e}}\right], \quad i=0,1, \ldots$.

We obtain the following remarkably simple result:

Theorem 3.2. Let $\Sigma(M, B, f)$ be a nonlinear system and let $b_{0} \in B$ be such that $f\left(b_{0}\right)=0$. Then there exist fibre respecting coordinates $(x, u)$ around $b_{0}$ such that $\Sigma(M, B, f)$ has the controllable linear representation

$\dot{x}=A x+B u$

(i.e. $f(x, u)=A x+B u$, with $A$ and $B$ matrices) if and only if the distributions $\Delta_{i}^{\mathrm{e}}$ are regular around $b_{0}$ and

$\operatorname{dim} \Delta_{n}^{\mathrm{e}}\left(b_{0}\right)=\operatorname{dim} B$

(with $\operatorname{dim} M=n$ ).

Remark 1. If the system has the representation

$\dot{\tilde{x}}=f(\tilde{x}, \tilde{u})$, with $f(0,0)=0$,

then this amounts to the existence of a feedback $\tilde{u}=\alpha(\tilde{x}, u)$ and a coordinate transformation $\tilde{x}=$ $\phi(x)$, with $\alpha(0,0)=0$ and $\phi(0)=0$, such that $\dot{\tilde{x}}=$ $f(\tilde{x}, \tilde{u})$ is transformed into $\dot{x}=A x+B u$.

Remark 2. The condition $\operatorname{dim} \Delta_{n}^{\mathrm{e}}\left(b_{0}\right)=\operatorname{dim} B$ is equivalent to the strong accessibility of the extended system as well as the strong accessibility of $\Sigma(M, B, f)$ around $b_{0}$, cf. $[12,13]$. (Note that $\Delta_{k}^{\mathrm{e}}\left(b_{0}\right)=\Delta_{n}^{\mathrm{e}}\left(b_{0}\right), k \geqslant n$, if $\Delta_{n}^{\mathrm{e}}$ is regular.)

Remark 3. It follows that $\Sigma(M, B, f)$ is linearizable if and only if its extended system is linearizable (see Theorem 3.1). 
Proof. The 'only if' direction is trivial. For the 'if' direction we notice that since $\Delta_{0}^{\mathrm{e}} \subset \Delta_{i}^{\mathrm{e}}$ the projections $\pi_{*} \Delta_{i}^{\mathrm{e}}$ are regular distributions on $M$ (all our statements will be local around $b_{0}$ or $\left.\pi\left(b_{0}\right)\right)$. Denote

$D_{i}=\pi_{*} \Delta_{i}^{\mathrm{e}}, \quad i=1, \ldots, n$.

Since $\operatorname{dim} \Delta_{n}^{\mathrm{e}}\left(b_{0}\right)=\operatorname{dim} B$ there exists an $N \leqslant n$ such that $\operatorname{dim} D_{N}=n$. Therefore the sequence $D_{1}$ $\subset D_{2} \subset \cdots \subset D_{N}$ satisfies the condition of Lemma 1 in [4]. Hence around $x_{0}=\pi\left(b_{0}\right)$ there exists a coordinate system $x=\left(x_{1}, \ldots, x_{n}\right)$ such that the integral manifolds of $D_{i}$ around $x_{0}$ are of the form $x_{i}=C_{i}, \quad i=\mu_{j}+1, \ldots, n, \quad C_{i}$ constant

with $\operatorname{dim} D_{i}=\mu_{i}$. Denote $x=\left(x^{1}, \ldots, x^{N}\right)$ where $x_{1}$ consists of the first $p_{1}=\mu_{1}$ coordinates of $x, x^{2}$ consists of the next $p_{2}=\mu_{2}-\mu_{1}$ coordinates, $\ldots, x^{N}$ consists of the last $p_{N}=\mu_{N}-\mu_{N-1}$ coordinates of $x$. Let $(x, u)$ be fibre respecting coordinates of $B$ and write $f=\left(f^{1}, \ldots, f^{N}\right)$ corresponding to the splitting $x=\left(x^{1}, \ldots, x^{N}\right)$. Then the following basic facts follow from the proof of Theorem 1 in [4]:

(A) The component functions $f^{j}$ do not depend on the variables $x^{1}, \ldots, x^{j-2}$ for $j=3,4, \ldots, N$. Also the $f^{j}$ do not depend on $u$ for $j \geqslant 2$.

(B) Rank $\partial f^{j} / \partial x^{j-1}=p_{j}$ for $j \geqslant 2$ and rank $\partial f^{1} / \partial u=p_{1}$.

Now we exactly follow the procedure of Jakubczyk and Respondek and modify the coordinates $\left(x^{1}, \ldots, x^{N}\right)$ in such a way that the integral manifolds of $D_{i}$ remain constant. In the first step we define the new coordinates $y^{N-1}, y^{j}=x^{j}$ if $j \neq N-1$, in such a way that in the new $y$-coordinates

$f=\left(f^{1}, \ldots, f^{N-1}, \bar{y}^{N-1}\right)$,

where $\bar{y}^{N-1}$ are $p_{N}$ coordinates of $y^{N-1}$. This is possible since rank $\partial f^{N} / \partial x^{N-1}=p_{N}$, and $f^{N}$ does not depend on $u, x^{1}, \ldots, x^{N-2}$. Denoting the new coordinates again by $\left(x^{1}, \ldots, x^{N}\right)$, we introduce in the second step the new coordinates $y^{N-2}, y^{j}=x^{j}$, if $j \neq N-2$, in such a way that

$f=\left(f^{1}, \ldots, f^{N-2}, \bar{y}^{N-2}, \bar{x}^{N-1}\right)$,

where $\bar{y}^{N-2}$ are $p_{N-1}$ coordinates of $y^{N-2}$. This is again possible because of (A) and (B). So we proceed $N-1$ steps till we obtain

$f=\left(f^{1}, \bar{x}^{1}, \ldots, \bar{x}^{N-1}\right)$.

Finally by (B) we can choose new input coordinates $\tilde{u}$ such that

$f=\left(\overline{\tilde{u}}, \bar{x}^{1}, \ldots, \bar{x}^{N-1}\right)$,

where $\overline{\tilde{u}}$ are $p_{1}$ coordinates of $\tilde{u}$. The relation between the old coordinates $u$ and the new coordinates $\tilde{u}$ is given by a feedback $u=\alpha(x, \tilde{u})$. Therefore in the new fibre respecting coordinates $(x, \bar{u})$ we obtain the linear system $\dot{x}=A x+B \tilde{u}$, with $(A, B)$ in a (slightly modified) Brunovsky form.

Remark. Notice that the adaptation of the $x$-coordinates in every step of the Jakubczyk-Respondek procedure can also be understood in terms of applying feedback! In fact in the $i$-th step the new coordinate functions $y^{N-i}$ are defined by

$y^{N-i}=\left(f^{N-i+1}, \bar{x}^{N-i}\right)$,

where $\bar{x}^{N-i}$ denotes $p_{N-i}-p_{N-i+1}$ coordinates of $x^{N-i}$ chosen in such a way that

$\operatorname{rank} \frac{\partial y^{N-i}}{\partial x^{N-i}}=p_{N-i}$

(cf. [4]). Now since by (A), $f^{N-i+1}$ does not depend on the variables $u, x^{1}, \ldots, x^{N-i+1}$, the relation between $y^{N-i}$ and $x^{N-i}$ is of the following form:

$y^{N-i}=\alpha\left(x^{N-i+1}, \ldots, x^{N}, x^{N-i}\right)$.

By interpreting $\left(x^{N-i+1}, \ldots, x^{N}\right)$ as the state variables this constitutes a nonlinear feedback transforming the old 'input' coordinates $x^{N-i}$ into the new ones $y^{N-i}$.

\section{Input-output decoupling}

The input-output decoupling problem with state feedback for general nonlinear systems can be formulated as follows. Consider a system with $m$ inputs $u=\left(u_{1}, \ldots, u_{m}\right)$

$\dot{x}=f(x, u)$

together with $m$ output maps

$y_{i}=h_{i}(x, u), \quad i=1, \ldots, m$,

where $h_{i}: B \rightarrow N_{i}$ is a smooth map from $B$ to a 
smooth $p_{i}$-dimensional manifold $N_{i}$. We now seek for a feedback

$u=\alpha(x, \tilde{u}), \quad \tilde{u}=\left(\tilde{u}_{1}, \ldots, \tilde{u}_{m}\right)$,

such that in the modified system

$\dot{x}=\tilde{f}(x, \tilde{u})$,

$y_{i}=\tilde{h}(x, \tilde{u}), \quad i=1, \ldots, m$,

with

$f(x, \alpha(x, \tilde{u}))=\tilde{f}(x, \tilde{u})$

and

$h_{i}(x, \alpha(x, \tilde{u}))=\tilde{h}_{i}(x, \tilde{u})$,

the input $\tilde{u}_{i}$ docs not affect the output $y_{j}$ for $j \neq i$. Moreover we require the input $\bar{u}_{i}$ to 'control' the output $y_{i}, i=1, \ldots, m$ (cf. [3,9]). In order to avoid not well posed problems we will require throughout that the output maps are independent, i.e.

$\operatorname{rank}\left(h_{1}, \ldots, h_{m}\right)=p$

with $p=p_{1}+\cdots+p_{m}$.

Recently the above problem was solved for affine systems

$\dot{x}=A(x)+\sum_{i=1}^{m} u_{i} B_{i}(x)$

with output maps $y_{i}=C_{i}(x)$ only depending on the state [9]. In fact our Theorem 4.2 will only be a generalization of the results obtained in [9].

Again the trick will be to consider the extended system

$\dot{x}=f(x, u), \quad \dot{u}=v$,

with the output maps (now only depending on the state $(x, u))$

$y_{i}=h_{i}(x, u), \quad i=1, \ldots, m$,

and to solve the input-output decoupling problem for this affine system.

Recall the definition of a controllability distribution for an affine system $\left(\Delta, \Delta_{0}\right)$ (see [6], also [5]). A regular distribution $R$, such that $\Delta_{0} \cap R$ has constant dimension, is a controllability distribution if

(1) $[\Delta, R] \subset R+\Delta_{0}$ (i.e. $R$ is locally controlled invariant),

(2) there does not exist a regular distribution $R^{\prime} \subset R$ such that $R^{\prime} \cap \Delta_{0}=R \cap \Delta_{0}$ and $\left[\Delta, R^{\prime}\right] \subset$ $R^{\prime}+\Delta_{0}$.
Remark: In the terminology of [6] these are the regular controllability distributions.

Let now $R^{e}$ be a controllability distribution of an extended system $\left(\Delta^{\mathrm{e}}, \Delta_{0}^{\mathrm{e}}\right)$. Then since $\left[\Delta_{0}, R^{\mathrm{e}}\right]$ $\subset R^{\mathrm{e}}+\Delta_{0}, R^{\mathrm{e}}$ projects to a regular distribution $R$ on $M$ [12]. Motivated by this we give the following definition of a controllability distribution for a general nonlinear system $\Sigma(M, B, f)$.

Definition 4.1. A regular distribution $R$ on $M$ such that $f_{*}^{-1} \dot{R} \cap \Delta_{0}^{\mathrm{c}}$ has constant dimension, is a controllability distribution for $\Sigma(M, B, f)$ if

(1) $f_{*}\left(\pi_{*}^{-1}(R)\right) \subset \dot{R}+f_{*}\left(\Delta_{0}^{\mathrm{e}}\right)$ (i.e. $R$ is locally controlled invariant),

(2) there does not exist a regular distribution $R^{\prime} \subset R$ such that

$f_{*}^{1} \dot{R}^{\prime} \cap \Delta_{0}^{\mathrm{e}}=f_{*}^{-1} \dot{R} \cap \Delta_{0}^{\mathrm{e}}$

and

$f_{*}\left(\pi_{*}^{-1}\left(R^{\prime}\right)\right) \subset \dot{R}^{\prime}+f_{*}\left(\Delta_{0}^{e}\right)$.

Remark. Recall that $\Sigma(M, B, f)$ is strongly accessible if there does not exist a regular distribution $D \neq T M$ such that $f_{*}\left(\pi_{*}^{-1}(D)\right) \subset \dot{D}$ (cf. $[12,13]$ ). So the above definition implies, as it should be, that the system 'restricted' to a controllability distribution is strongly accessible (cf. [6]).

Consequently, if $R$ is a controllability distribution for $\Sigma(M, B, f)$ then $f^{-1}(\dot{R})$ is a controllability distribution for the extended system $\left(\Delta^{\mathrm{e}}, \Delta_{0}^{\mathrm{e}}\right)$. Let us now denote by $R_{i}^{\text {e* }}$ the maximal controllability distribution (with respect to the extended system) contained in

$\bigcap_{j \neq i} \operatorname{Ker} h_{j *}$

( $R_{i}^{\mathrm{e} *}$ exists, see $[5,6]$ ).

We will make two standing assumptions:

(A1) $R_{i}^{\mathrm{e} *}$ has constant dimension, $\forall i$,

(A2) $R_{i}^{\mathrm{e} *} \cap \Delta_{0}^{\mathrm{e}}$ has constant dimension, $\forall i$.

(Or said in another way we will restrict ourselves to open subsets of $B$ where the above assumptions hold.) Then $R_{i}^{\text {e* }}$ will be regular distributions on $B$, and $R_{i}^{*}:=\pi_{*} R_{i}^{\mathrm{e}}, i=1, \ldots, m$, will be regular distributions on $M$. We will call $R_{i}^{*}$ the maximal controllability distribution (w.r.t. $\Sigma(M, B, f)$ 'contained in $\bigcap_{j+i} \operatorname{Ker} h_{j *}$ '. The main theorem reads now as follows. 
Theorem 4.2. Let $\Sigma(M, B, f)$ be a strongly accessible system with output maps

$h_{i}: B \rightarrow N_{i}, \quad i=1, \ldots, m$,

such that

$\operatorname{rank}\left(h_{1}, \ldots, h_{m}\right)=\operatorname{dim}\left(N_{1} \times \cdots \times N_{m}\right)$.

Suppose that assumptions (A1) and (A2) hold and that $\sum_{i \in I} R_{i}^{\mathrm{e} *}$ has constant dimension for every subset $I$ of $\{1, \ldots, m\}$. Then the input-output decoupling problem with static feedback can be locally solved if and only if

$\Delta_{0}^{\mathrm{e}}=\Delta_{0}^{\mathrm{e}} \cap R_{1}^{\mathrm{e} *}+\cdots+\Delta_{0}^{\mathrm{e}} \cap R_{m}^{\mathrm{e} *}$.

Remark 1. Condition (4.4) is equivalent to the solution of the input-output decoupling problem for the extended system, see [9].

Remark 2. Consider the statements:

(A) $\sum_{i \in I} R_{i}^{\mathrm{e} *}$ has constant dimension, $\forall I$,

(B) $\sum_{i \in I} R_{i}^{*}$ has constant dimension, $\forall I$.

It is clear that, under the standing assumption (A2):
(B) $+(4.4) \Rightarrow(A)$ and
(A) $+(4.4) \Rightarrow(B)$

Remark 3. One can give examples where assumptions (A1) or (A2) are not satisfied but input-output decoupling still can be achieved. In this sense Theorem 4.2 only gives 'sufficient' conditions for input-output decoupling.

Proof. We closely follow the proof of Theorem 3.1 in [9]. An essential step is that condition (4.4) implies that for every $I \subset\{1, \ldots, m\}$ the distributions $\sum_{i \in I} R_{i}^{\text {e** }}$ are involutive and hence regular. This yields that for every $I, \sum_{i \in I} R_{i}^{\text {e** }}$ is a regular controllability distribution w.r.t. $\left(\Delta^{\mathrm{e}}, \Delta_{0}^{\mathrm{e}}\right)$, with

$$
\sum_{i \in I} R_{i}^{\mathrm{e} *} \cap \Delta_{0}^{\mathrm{e}}
$$

of constant dimension.

Hence $\sum_{i \in I} R_{i}^{*}$ is for every $I$ a regular controllability distribution w.r.t. $\Sigma(M, B, f)$. We are now going to construct the feedback $u=\alpha(x, \tilde{u})$ which will make every $R_{i}^{*}$ invariant, i.e.

$\left[\tilde{f}(\cdot, \tilde{u}), R_{i}^{*}\right] \subset R_{i}^{*}, \quad \forall \tilde{u}, i=1, \ldots, m$.
Since $\sum_{j \star i} R_{i}^{\mathrm{e} *}$ is regular and by (4.4)

$\operatorname{dim}\left(\sum_{j \neq i} R_{i}^{\mathrm{e} *} \cap \Delta_{0}^{\mathrm{e}}\right)=m-1$,

we can (locally) construct an $(n+m-1)$ dimensional regular distribution $E_{i}$ on $B$ such that

$\sum_{j \neq i} R_{i}^{\mathrm{e} *} \subset E_{i}$ and $\pi_{*} E_{i}=T M$.

This we can do for every $i=1, \ldots, m$. Now it is clear from (4.4) that $E:=\bigcap_{i=1}^{m} E_{i}$ is again a regular distribution satisfying $\pi_{*} E=T M$. In fact $\operatorname{dim} E$ $=\operatorname{dim} M$, so $E$ is (locally) a horizontal regular distribution on $B$ (cf. [7]), or said in another way, an integrable connection (locally) on $B$. The required feedback $u=\alpha(x, \tilde{u})$ is now basically determined by the following condition [7]: The sections $\tilde{u}_{i}=C_{i}\left(C_{i}\right.$ constants $)$ are the integral manifolds of $E$.

In such new fibre respecting coordinates $(x, \bar{u})$, the distribution $R_{i}^{*}$ on $M$ can be trivially lifted to distributions on $B$, also denoted by $R_{i}^{*}$, by requiring that $R_{i}^{*} \subset E$. The distributions $R_{i}^{\mathrm{e} *}$ are then given (possibly after renumbering of $\tilde{u}_{1}, \ldots, \tilde{u}_{m}$ ) by

$R_{i}^{\mathrm{e} *}=R_{i}^{*}+\operatorname{rank}\left\{\frac{\partial}{\partial \tilde{u}_{i}}\right\}, \quad i=1, \ldots, m$.

Since $\Delta_{0}^{\mathrm{e}} \subset \sum_{i=1}^{m} R_{i}^{\mathrm{e}^{*}}$ and the system is strongly accessible it follows that

$\sum_{i=1}^{m} R_{i}^{\mathrm{e} *}=T B$ and $\sum_{i=1}^{m} R_{i}^{*}=T M$.

Hence

$\left(h_{1}, \ldots, h_{m}\right)_{*} \sum_{i=1}^{m} R_{i}^{\mathrm{e} *}=T\left(N_{1} \times \cdots \times N_{m}\right)$

and $h_{i *} R_{i}^{\mathrm{e} *}=T N_{i}$ for every $i=1, \ldots, m$. This means that the $i$-th input $\tilde{u}_{i}$ controls the $i$-th output $y_{i}$.

Remark 1. The required coordinates $\left(\tilde{u}_{1}, \ldots, \tilde{u}_{m}\right)$, or the integrable connection $E$ are basically constructed as follows. Each distribution $\sum_{i \neq i} R_{j}^{\mathrm{e} *}$ determines the connection in the $\partial / \partial \tilde{u}_{i}$-direction. Hence together they determine the total connection.

Remark 2. Analogous to [10] the above theorem is easily extended to the case of more inputs than output maps. 


\section{Acknowledgements}

It is a pleasure to thank Henk Nijmeijer for some stimulating discussions, especially about the input - output decoupling problem.

\section{References}

[1] R.W. Hirschorn, $(A, B)$-invariant distributions and disturbance decoupling of nonlinear systems, SIAM J. Control Optim. 19 (1981) 1-19.

[2] L.R. Hunt and R. Su, Linear equivalents of nonlinear time varying systems, Int. Symposium on Math. Theory of Networks and Systems (1981) pp. 119-123.

[3] A. Isidori, A.J. Krener, C. Gori-Giorgi and S. Monaco, Nonlinear decoupling via feedback: A differential geometric approach, IEEE Trans. Automat. Control 26 (1981) 331-345.

[4] B. Jakubczyk and W. Respondek, On linearization of control systems, Bull Acad. Pol. Sci. Ser. Sci. Math 28 (1980) 517-522.

[5] A.J. Krener and A. Isidori, (Ad $f, g$ ) invariant and controllability distributions, in: Feedback Control of Linear and Nonlinear Systems, Lect. Notes in Control and Inform. Sci. Vol. 39 (Springer, Berlin-New York, 1982).
[6] H. Nijmeijer, Controllability distributions for nonlinear systems, Systems Control Lett. 2 (1982) 122-129.

[7] H. Nijmeijer and A.J. van der Schaft, Controlled invariance for nonlinear systems, IEEE Trans. Automat. Control 27 (1982) 904-914.

[8] H. Nijmeijer and A.J. van der Schaft, The disturbance decoupling problem for nonlinear control systems, IEEE Trans. Automat. Control 28 (1983) 621-623.

[9] H. Nijmeijer and J.M. Schumacher, The noninteracting control problem for nonlinear control systems, Memo No. 427, Dept. Appl. Math., Twente University of Technology (1983).

[10] H. Nijmeijer and J.M. Schumacher, Zeros at infinity for affine nonlinear control systems, Memo No. 441, Twente University of Technology (1983).

[11] H. Nijmeijer and J. M. Schumacher, Input-output decoupling of nonlinear systems with an application to robotics, Memo No. 444, Twente University of Technology (1983).

[12] A.J. van der Schaft, Observability and controllability for smooth nonlinear systems, SIAM Journal Control Optim. 20 (1982) 338-354.

[13] A.J. van der Schaft, System theoretic descriptions of physical systems, Doct. Diss., Groningen 1983, CWI Tracts 3 (Amsterdam, 1984).

[14] R. Su, On the linear equivalents of nonlinear systems, Systems Control Lett. 2 (1982) 48-52. 